\title{
Thermal Design of Power Transformers via CFD
}

\author{
Ralf Wittmaack \\ Siemens AG, EM TR LPT GTC RES PN, Katzwanger Str. 150, Nürnberg 90461, Germany
}

Received: August 18, 2014 / Accepted: September 30, 2014 / Published: January 31, 2015.

\begin{abstract}
At Siemens, an in-house CFD (computational fluid dynamics) code UniFlow is used to investigate fluid flow and heat transfer in oil-immersed and dry-type transformers, as well as transformer components like windings, cores, tank walls, and radiators. This paper outlines its physical models and numerical solution methods. Furthermore, for oil-immersed transformers, it presents an application to a HV (high voltage) winding in a traction transformer of locomotives, cooled by synthetic ester.
\end{abstract}

Key words: Thermal design, CFD, physical models, numerical methods.

\section{Introduction}

The life time of power transformers is substantially influenced by chemical degradation processes occurring in the electrical insulation. Since the speed of these processes depends significantly on temperature, the proper prediction of component temperatures during transformer operation is a crucial part of the design process. There are several sources of heat in the transformer. If a time varying voltage is applied, magnetic hysteresis effects and eddy currents lead to no-load losses in the steel sheets of the core. In addition, during normal operation, the electrical currents cause ohmic and stray load losses.

To keep the temperatures of the transformer components within acceptable limits, appropriate cooling is essential. Depending on the type of transformer, this is normally accomplished via natural or forced convection of the cooling fluids air or oil. In addition to mineral and silicone oil, natural and synthetic ester fluids are also used.

Thanks to its flexibility and accuracy, CFD (computational fluid dynamics) is increasingly being used to analyse transformer thermal design. This follows the trend established in other branches of

Corresponding author: Ralf Wittmaack, Dr.-Ing., research fields: CFD code development and application on nuclear safety, gas turbine development, aerospace research, and electrical transformers. E-mail: ralf.wittmaack@siemens.com. advanced technology development like aerospace, automotive, and power generation, where CFD simulations are indispensable parts of the product development cycles.

Employing commercial CFD codes, several detailed studies of disc-type transformer windings were performed by Torriano et al. [1] and Jiao [2]. Moreover, extended full geometry CFD analyses coupled to electromagnetic simulation of the load and no-load losses in core and windings were presented by Smolka et al. $[3,4]$.

Furthermore, combined oil and air flows in distribution transformers were investigated with commercial CFD codes by Fonte et al. [5] and Gastelluritia et al. [6].

Our intention is to provide a simulation method that may be used for detailed CFD analyses on fine grids as well as for simplified coarse grid studies. The in-house code UniFlow is designed to be applicable also by users with limited experience in CFD. For this reason, e.g., material attributes are employed for a convenient coupling of fluid and solid regions in conjugate heat transfer simulations.

\section{Physical Models and Numerical Methods}

\subsection{Physical Models}

Our physical model is aimed at investigating flows 
with several kinds of heat transfer in a complex geometry. It simulates the flow of single-component, incompressible Newtonian fluids in a three-dimensional geometry. In addition to the fluids, in gaseous or liquid state, several structural materials are considered as hydrodynamic obstacles and thermodynamic heat structures. The hydrodynamics is described by the continuity equation and the Navier-Stokes equation. For the simulation of turbulence, the algebraic eddy viscosity model of Baldwin and Lomax [7] is available. To simulate the transition between laminar and turbulent flows, algebraic transition models of Drela [8] and Mayle [9] are on hand.

For temperature dependent density or material properties of the viscous stress tensor, the hydrodynamics of the fluid is coupled to the thermodynamics. For this reason, internal heat transfer (by convection and conduction) and heat generation by internal sources as well as heat transfer to the surroundings are modelled via a heat transport equation. To allow for the simulation of phase transitions, it is provided in enthalpy formulation. At the rigid boundaries, heat conduction is considered. For coarse grids, convective heat transfer coefficients may be employed at solid-liquid interfaces. Radiant heat transfer is simulated at structural material surfaces. The material properties (density, dynamic viscosity, specific heat, heat conductivity, and convective heat transfer coefficient) depend on the temperature. Solids may have orthotropic heat conductivity.

\subsubsection{Dynamic Equations}

Our dynamic equations are written in Cartesian coordinates. The continuity equation for incompressible flow is:

$$
\frac{\partial}{\partial x^{m}}\left(\rho v^{m}\right)=0
$$

According to Landau and Lifshitz [10], where $\rho$ is density and $v$ velocity, $x$ is the space coordinates and we use Einstein's summation convention for the space direction index $m$. Introduction of the continuity equation into the Navier-Stokes equation [10] leads to a momentum equation in strong conservation form:

$$
\rho \frac{\partial v_{i}}{\partial t}+\frac{\partial}{\partial x^{m}}\left[\rho v_{i} v^{m}-\mu\left(\frac{\partial v_{i}}{\partial x_{m}}+\frac{\partial v^{m}}{\partial x^{i}}\right)\right]=-\frac{\partial p}{\partial x^{i}}+\rho g_{i}
$$

where, $t$ is time, $p$ pressure, and $g$ gravitational acceleration. After inclusion of the continuity equation, our heat transport equation in strong conservation form reads:

$$
\rho \frac{\partial h}{\partial t}+\frac{\partial}{\partial x^{m}}\left(\rho h v^{m}-\lambda \frac{\partial T}{\partial x_{m}}\right)=P_{d}
$$

Here, $h$ is specific enthalpy, $T$ temperature, $\lambda$ heat conductivity, and $P_{d}$ density of the heat sources or sinks.

\subsubsection{Radiant Heat Transfer Model}

Radiant heat transfer may be simulated between structural material surfaces adjacent to the fluid. The employed radiation model assumes that the radiating surfaces are boundaries of a hollow space with linear dimension much greater than their distance. It is applicable for, e.g., parallel plates and concentric cylinders. With this simplifying assumption, the power received by surface "a" via the heat transfer from surface " $b$ " is:

$$
P_{a b}=c_{a b} A_{a}\left(T_{b}^{4}-T_{a}^{4}\right)
$$

According to Baehr and Stephan [11], where,

$$
c_{a b}=\frac{\sigma}{\frac{1}{\varepsilon_{a}}+\frac{A_{a}}{A_{b}}\left(\frac{1}{\varepsilon_{b}}-1\right)}
$$

Here, $A$ is area of a radiating structural material surface, $T$ surface temperature, $\sigma=5.67051 \times 10^{-8}$ $\mathrm{W} /\left(\mathrm{m}^{2} \cdot \mathrm{K}^{4}\right)$ Stefan-Boltzmann constant, and $\varepsilon$ emissivity of a structural material surface. Computation domain nodes undergoing radiant heat transfer may have their radiation partner nodes inside the computation domain or at the boundary.

\subsection{Numerical Methods}

For the numerical representation of our model, we 
developed a finite volume method and employ boundary fitted, curvilinear, non-orthogonal, block-structured grids. The blocks may be connected via one-to-one or patched couplings. The arrangement of the dynamic variables in the control volumes of the grid is collocated at the node centre. The dynamic equations are solved sequentially. For the solution of the momentum, pressure-correction, and heat transport equations, we use implicit schemes. The system of continuity and momentum equations is solved by a SIMPLE [12], SIMPLEC [13], or PISO algorithm [14].

To speed up the code execution and to simplify the estimation of discretisation errors, a FAS (full approximation scheme) multi-grid algorithm is employed [15]. It is a geometric approach with standard coarsening applied to the outer iterations, visiting the grid levels in V-cycles. For steady-state problems, it operates as a FMG (full multi-grid) algorithm, whereas for transient problems, the algorithm starts at the finest grid.

For the efficient solution of sparse linear equations, several algorithms are available. The parabolic momentum and heat transport equations may be solved with SIP (strongly implicit procedure) solvers that are modified to handle block couplings via the residual vector, as outlined by Ferziger and Peric [12]. Additionally, for the elliptic pressure-correction equation, an aggregation-based algebraic multi-grid algorithm of Notay [16] is available.

\section{Application}

In this section, we describe an investigation of a $\mathrm{HV}$ (high voltage) winding of a locomotive transformer of high speed trains. The goal is to find the maximum temperatures in the insulation materials to allow for the selection of appropriate materials that withstand the thermal load. To cool the winding, a pump driven flow of synthetic ester is used. Figs. 1 and 2 show an outside view with removed top cover and the active part of the transformer without tap changer.

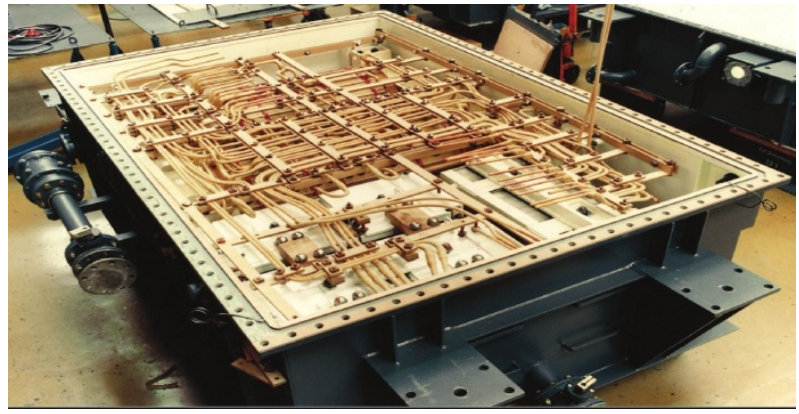

Fig. 1 Outside view of transformer without top cover.

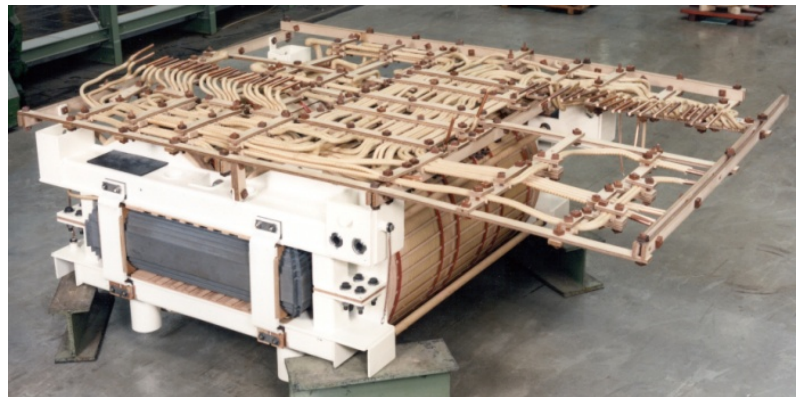

Fig. 2 Active part of transformer without tap changer.

\subsection{Geometry Model}

The single phase transformer has two limbs to make it more compact. We analyze one of the limbs and consider a periodic three-dimensional $7.5^{\circ}$ segment of the circumference. The periodicity comes from 24 spacers along the circumference. Fig. 3 shows the initial specific enthalpy of an axial part of the angular segment of the winding. It explains the location of the materials: grey indicates ester, red pressboard, blue conductor, and light blue Nomex. This axial part consists of half a coil from the 92 coils in total.
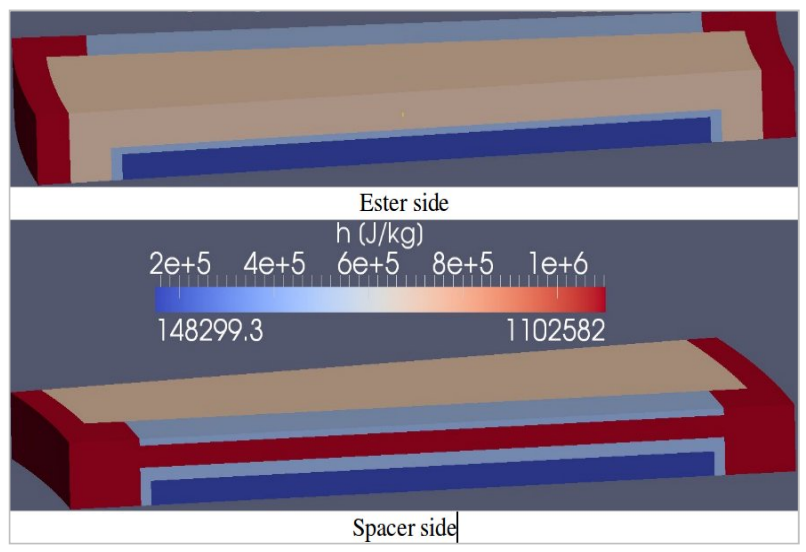

Fig. 3 Location of materials in axial part of circumferential winding segment. 


\subsection{Grid}

A single block hexahedral grid with 706,560 nodes is used and 222,880 nodes represent the ester while the rest correspond to structural materials. The node lengths are in the range of $0.62 \mathrm{~mm}$ to $5.1 \mathrm{~mm}$.

\subsection{Boundary Conditions}

At the inlet, the pump driven oil flow occurs with a velocity of $0.9 \mathrm{~m} / \mathrm{s}$ and a temperature of $364.55 \mathrm{~K}$. All computation domain boundaries are adiabatic, except the inlet and outlet.

\subsection{Material Properties}

Structural materials considered in this simulation are copper and its adjacent Nomex 410 isolation as well as pressboard and Nomex 994 isolation. In our grid, the winding is split up into an inner and an outer part, made up of the copper conductor and Nomex 410. This is aimed at separating the inner part that consists mainly of $\mathrm{Cu}$, from the outer region, where the dominant part of the Nomex 410 insulation is located. Their mixture material properties are calculated via:

$$
\begin{array}{r}
\rho_{\text {mix }}=\sum_{i=1}^{n_{\text {mat }}} \alpha_{i} \rho_{i}, c_{p \text { mix }}=\sum_{i=1}^{n_{\text {mat }}} x_{i} c_{p i}, \\
\lambda_{\text {mix }}=\left(\sum_{i=1}^{n_{\text {mat }}} \frac{\alpha_{i}}{\lambda_{i}}\right)^{-1}, \alpha_{i}:=\frac{V_{i}}{V}, x_{i}:=\alpha_{i} \frac{\rho_{i}}{\rho_{\text {mix }}}(6)
\end{array}
$$

Here $n_{\text {mat }}$ is no. if considered structural materials, $V$ volume, $c_{p}$ specific heat at constant pressure, $\alpha$ volume fraction, and $x$ mass fraction.

Several modes of operation exist in the transformer, e.g., rated current and overload. In our simulation, we consider rated current that corresponds to an average power density of the load losses $P_{d}=1.389 \mathrm{MW} / \mathrm{m}^{3}$. The load losses are calculated by a Maxwell solver and subsequently mapped to the CFD grid.

\subsection{Non-dimensional Numbers and Boundary Layer Thickness}

With the width of a winding segment of $l=74.5 \mathrm{~mm}$ as characteristic length, the ester inlet velocity of 0.9 $\mathrm{m} / \mathrm{s}$, and the average ester temperature of $368 \mathrm{~K}$, the Reynolds number $R$ is 6,875 and the Prandtl number is 140 . The ester flow along a winding segment resembles flow along a flat plate, where the transition from laminar to turbulent flow occurs between $R=3.5 \times 10^{5}$ and $3.5 \times 10^{6}$, according to Ref. [17]. This indicates that the ester flow is laminar, i.e., the hydrodynamic and thermal boundary layer thickness may be estimated according to Ref. [10]:

$$
\delta_{h} \propto \frac{l}{\sqrt{R}} ; \delta_{t} \propto \delta_{h} \operatorname{Pr}^{-\frac{1}{3}}
$$

This leads to $\delta_{h}=0.9 \mathrm{~mm}$ and $\delta_{t}=0.17 \mathrm{~mm}$ at the end of a winding segment, i.e., the grid of our simulation of the traction transformer windings is too coarse to resolve the thermal boundary layer. To compensate for that, convective heat transfer coefficients are used at all interfaces of ester and structural materials. These were calculated via a detail model and a fine grid.

\subsection{Simulation Results}

As a result of the axial flow barriers, there is a meandering oil flow in the radial channels. The maximum radial velocity component is similar to the inlet velocity, while the maximum vertical velocity is considerably higher than the inlet velocity. The maximum value occurs at the inner side, where the flow area is smaller. Figs. 4 and 5 show the radial and axial velocity components.

Fig. 6 shows the calculated pressure in the ester. There is some stagnation pressure at the axial flow barriers. Since the limbs are oriented horizontally, we run our simulations without gravitational acceleration. For this reason, there is no hydrostatic contribution to the pressure.

The temperature of ester and structural materials is shown in Figs. 7 and 8, at the ester and the spacer side of the winding. It indicates that the maximum temperatures are encountered at the spacer side.

The maximum $\mathrm{Cu}$ temperature of $384.2 \mathrm{~K}$ is calculated at the spacer side of the windings. At that side of the HV windings, the temperature differs only 


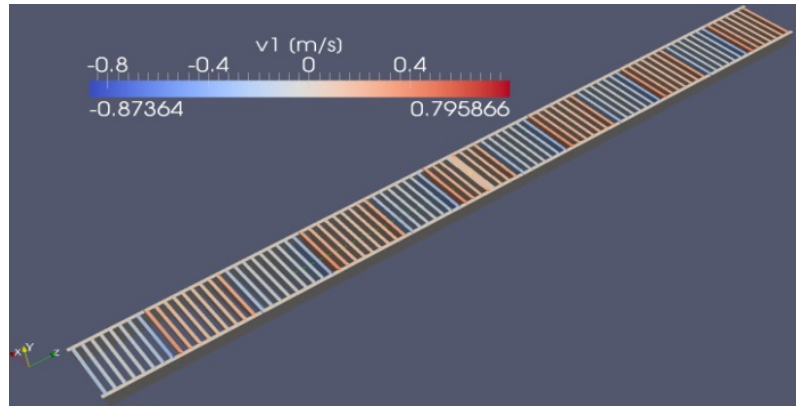

Fig. 4 Radial component of oil velocity.

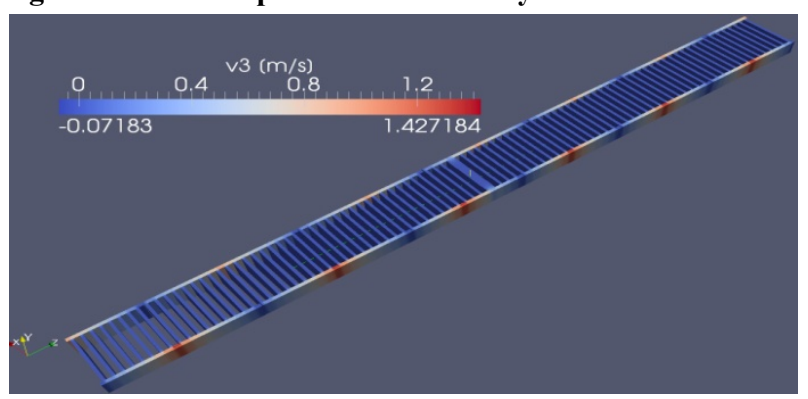

Fig. 5 Axial component of oil velocity.

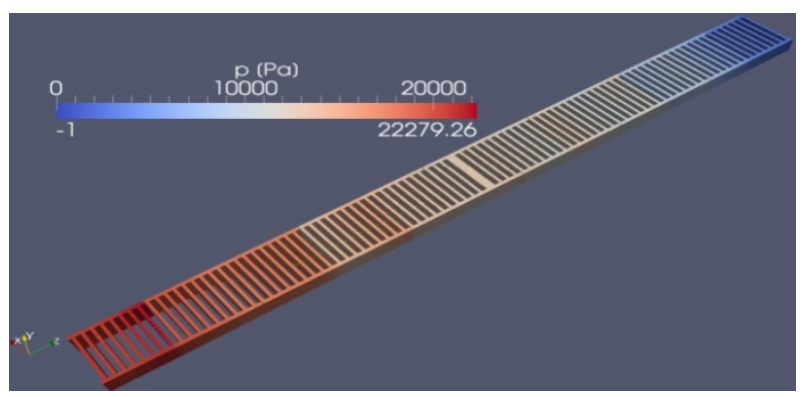

Fig. 6 Pressure of oil.

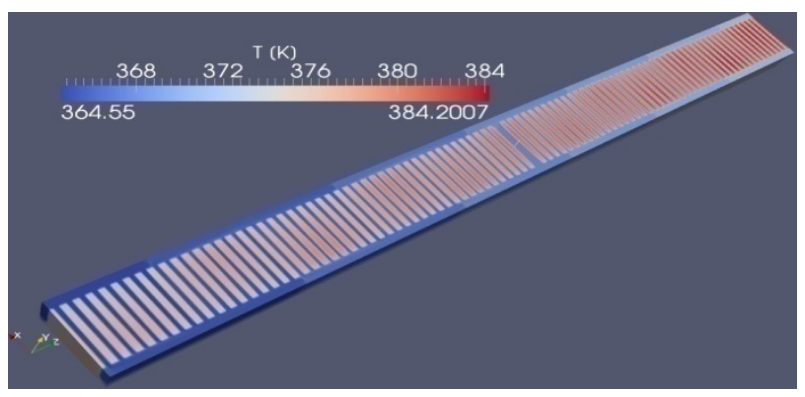

Fig. 7 Temperature at ester side of winding.

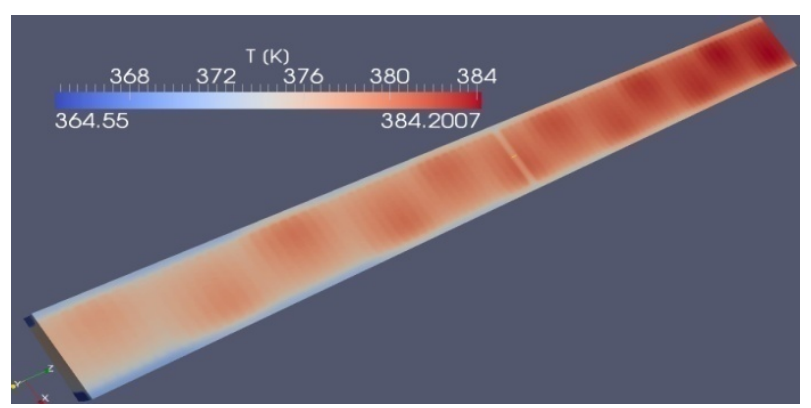

Fig. 8 Temperature at spacer side of winding. slightly between $\mathrm{Cu}$ and the adjacent insulation materials Nomex 994 and pressboard.

The maximum temperature of the structural materials is also very close. The maximum temperature in the Nomex 944 of $384.14 \mathrm{~K}$ and in the pressboard of $384.09 \mathrm{~K}$ are particularly interesting.

According to Ref. [18], enhanced thermal ageing and cracking of the ester may occur at temperatures above $403 \mathrm{~K}$. The maximum temperature of ester in the simulation is more than $27 \mathrm{~K}$ below this threshold.

\section{Conclusions}

We analysed the thermal design of the HV windings of a traction transformer in the steady state with the CFD code UniFlow. This winding is cooled by synthetic ester in the OD (oil direct) mode.

In addition to the result shown here, the thermal design of cast resin transformers can be studied, including radiant heat transfer between core, windings, and radiation cylinders. This is demonstrated in Ref. [19]. Other applications are related to detailed analyses on segments of disc windings with respect to, e.g., modelling of material compositions, width of oil channels. Another field of application is oil flows in transformer cores. Moreover, combined oil and air flows are analysed in the context of fin type distribution transformers. This is aimed at optimisation of the thermal efficiency of the fins and other tasks. Furthermore, combined oil and air flows in radiators can be investigated.

In addition to steady state analyses, transient processes are investigated. One interesting type of transient occurs at the cold start of a transformer. This matters in particular for oil transformers where the dynamic viscosity is very high at low temperatures, especially for ester fluids.

The future work will include validation of the calculated results via experimental data. The reason why this has not yet been accomplished is that detailed measurements inside power transformers are quite demanding, e.g., as a result of the high electric and magnetic fields. However, a comprehensive verification 
of UniFlow was performed via analytical solutions. This covers steady viscous flow between parallel plates and concentric cylinders, potential flow around a cylinder, transient heat conduction in a parallelepiped, as well as heat convection and heat-up by a source. Furthermore, the simulation of transitional air flow onto a flat plate was validated via experiments outlined by Schlichting [17].

\section{References}

[1] Torriano, F., Pichler, P., and Chaaban, M. 2012. "Numerical Investigation of 3D Flow and Thermal Effects in a Disc-type Transformer Winding." Applied Thermal Engineering 40: 121-31.

[2] Jiao, Y. 2012. "CFD Study on the Thermal Performance of Transformer Disc Windings without Oil Guides." M.Sc thesis, KTH School of Industrial Engineering and Management.

[3] Smolka, J., and Nowak, A. J. 2008. "Experimental Validation of the Coupled Fluid Flow, Heat Transfer and Electromagnetic Numerical Model of the Medium Power Dry-Type Electrical Transformer." International Journal of Thermal Sciences 47 (10): 1393-410.

[4] Smolka, J., Biro, O., and Nowak, A. J. 2009. "Numerical Simulation and Experimental Validation of Coupled Flow, Heat Transfer and Electromagnetic Problems in Electrical Transformers." Archives of Computational Methods in Engineering 16 (3): 319-55.

[5] Fonte, C. M., Campelo, H., Sousa, R. G., Dias, M. M., Lopes, J. C. B., and Lopes, R. 2011. "CFD Analysis of Core-Type Power Transformers." Presented at the 21st International Conference on Electrical Distribution, Frankfurt, Germany.

[6] Gastelluritia, J., Ramos, J. C., Larraona, G. S., Rivas, A., Izagirre, J., and del Río, L. 2011. "Numerical Modelling of
Natural Convection of Oil inside Distribution Transformers." Applied Thermal Engineering 31 (4): 493-505.

[7] Baldwin, B. S., and Lomax, H. 1978. "Thin Layer Approximation of and Algebraic Model for Separated Turbulent Flows." AIAA-paper, NO. 78-0257.

[8] Drela, M., 1998. MISES Implementation of Modified Abu-Ghannam/Shaw Transition Criterion, MIT Aero-Astro, Boston, MA, USA.

[9] Mayle, R. E. 1991. "The Role of Laminar-Turbulent Transition in Gas Turbine Engines." ASME Journal of Turbomachinery 113 (4): 509-36.

[10] Landau, L. D., and Lifshitz, E. M. 1989. "Fluid Mechanics." In Course of Theoretical Physics. Oxford: Pergamon Press.

[11] Baehr, H. D., and Stephan, K. 2006. Heat and Mass Transfer. Berlin: Springer-Verlag.

[12] Ferziger, J. H., and Peric, M. 1999. Computational Methods for Fluid Dynamics. Berlin: Springer-Verlag.

[13] van Doormal, J. P., and Raithby, G. D. 1984. "Enhancements of the SIMPLE Method for Predicting Incompressible Flows." Numerical Heat Transfer 7 (2): 147-63.

[14] Issa, R. I. 1986. "Solution of Implicitly Discretized Fluid Flow Equations by Operator Splitting." Journal of Computational Physics 62 (1): 40-65.

[15] Trottenberg, U., Oosterlee, C. W., and Schüller, A. 2001. Multigrid. New York: Academic Press.

[16] Notay, Y. 2010. "An Aggregation-Based Algebraic Multigrid Method." Electronic Transactions on Numerical Analysis 37: 123-46.

[17] Schlichting, H. 1979. Boundary-Layer Theory. New York: McGraw-Hill.

[18] Vosen, H. 1997. Kühlung und Belastbarkeit von Transformatoren. Berlin: VDE-Verlag GmbH.

[19] Wittmaack, R. 2014. "Thermal Design of Power Transformers via CFD." Presented at the 11th World Congress on Computational Mechanics, Barcelona, Spain. 